\title{
Measurement and Calculation of the Liquid-Liquid Phase Boundaries and Phase Equilibria for the Hexane+Polyethylene System at High Temperatures
}

\author{
Masashi Haruki, Shohei Fujikawa, Kotaro Adachi, Yuki Hadano, \\ Shin-ichi KiHARA and Shigeki TAKISHIMA \\ Department of Chemical Engineering, Graduate School of Engineering, Hiroshima University, \\ 1-4-1 Kagamiyama, Higashi-Hiroshima-shi, Hiroshima 739-8527, Japan
}

\begin{abstract}
Keywords: Polyethylene, Hexane, Cloud Point, Phase Equilibrium, Equation of State
The liquid-liquid phase equilibria for the hexane+polydisperse polyethylene (PE) systems were measured at $473 \mathrm{~K}$ and at feed PE weight fractions ranging from 0.075 to 0.20 . An apparatus was developed for the measurement, which consisted of a variable-volume optical cell and two sampling tubes for correcting both the top (hexane-rich) and bottom (PE-rich) phases. The PE weight fraction of the hexane-rich phase decreased with decreases in the feed weight fraction of $\mathrm{PE}$ and the phase equilibrium pressure. On the other hand, that of the PE-rich phase increased as the feed PE weight fraction and pressure decreased. Furthermore, the experimental data were compared with the predicted results obtained using the Sanchez-Lacombe equation of state. The predicted results approximately reproduced the experimental results for the PE weight fractions of the hexane-rich phase. Although some deviations were found in the experimental results of the PE-rich phase, the predicted phase equilibrium lines qualitatively reproduced the experimental results, which showed that the PE weight fraction increased with decreases in the feed PE weight fraction and pressure. Moreover, both the experimental and the predicted results also indicated that the influences of the feed PE weight fraction on the weight average molecular weight and on the polydispersity index of PE in the PE-rich phase were larger than those in the hexane-rich phase at low pressure.
\end{abstract}

\section{Introduction}

Polyethylene (PE) is widely utilized as an industrial polymer. Solution polymerization is effective for PE production because many olefin comonomers can be added to produce excellent characteristics. However, the process of separating products from the reaction media and from residual monomers requires much energy after polymerization. It is well known that the energy cost can be reduced by using a high-temperature liquid-liquid (LL) separator prior to an evaporator, and the separation temperature also can be controlled via the addition of low molecular weight substances such as the ethylene that is a monomer of PE (McClellan et al., 1985; Folie and Radosz, 1995). Therefore, knowledge of the LL phase separation behavior of the PE solution at high temperatures is imperative for the development and the operation of the separation process as well as a polymerization reactor. A great deal of experimental data on the LL phase boundaries of PE solutions, such as the solvent $+\mathrm{PE}$ and the gaseous substance + solvent $+\mathrm{PE}$ systems at high temperatures and pressures, has been reported thus far. For example, the effects of the molecular weight of $\mathrm{PE}$, the species of the

Received on November 4, 2015; accepted on December 2, 2015 DOI: $10.1252 /$ jcej. 15 we281

Correspondence concerning this article should be addressed to $\mathrm{M}$. Haruki (E-mail address: mharuki@hiroshima-u.ac.jp) or S. Takishima (E-mail address: r736735@hiroshima-u.ac.jp). solvent, and the concentration of the gaseous substance have been studied (McHugh and Guckes, 1985; Chen and Radosz, 1992; Kiran et al., 1993a, 1993b; Xiong and Kiran, 1994; de Loos et al., 1996; Chen et al., 2004; Nagy et al., 2006, 2007; Haruki et al., 2008, 2009, 2010).

A typical phase diagram for the solvent $+\mathrm{PE}$ system appears in Figure 1. As shown in the figure, the LL phase boundary curve (cloud point curve) exactly accords with the phase equilibrium lines (polymer-rich phase line and solvent-rich phase line) regardless of the feed PE concentration for the solvent + monodisperse polymer binary system. Therefore, the phase equilibrium data can be obtained from the cloud point measurements. However, the LL phase boundary curve does not correspond to the phase equilibrium curves for the solvent + polydisperse polymer system (Folie and Radosz, 1995). When the pressure of the solution that forms the homogeneous phase in the high-pressure cell is decreased gradually under a constant temperature and feed composition, a new phase is generated at the cloud point. The locus of the composition of the newly generated phase at the cloud point of each feed composition is called a shadow curve. We can obtain phase equilibria by additional depressurization of the solution in the cell, which shows different loci from the LL phase boundary curve, and a phase equilibrium depends on the feed PE concentration as well as on the temperature and pressure, as shown in Figure 1. Since PEs that are produced industrially often have polydispersity, the phase equilibrium data for the PE solutions are, 

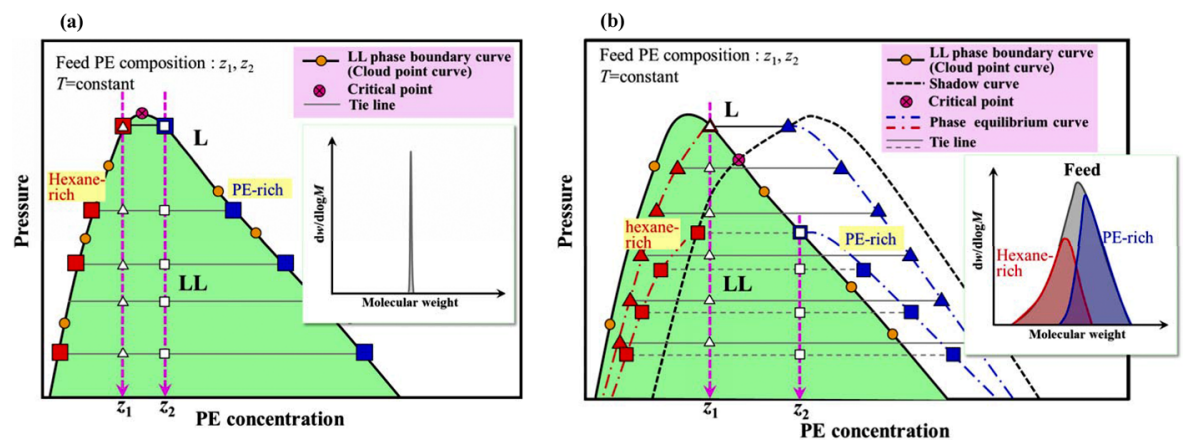

Fig. 1 Typical phase diagram of the solvent + PE system: (a) solvent + monodisperse PE: (b) solvent + polydisperse PE

therefore, as important as the phase boundary data for the process design of the solution polymerization of $\mathrm{PE}$ at high levels of temperature and pressure.

The phase equilibria of the polymer solutions at high pressure have been the focus of a few studies. Seckner et al. (1988) measured the phase equilibria for the polystyrene + toluene + ethane system at $70^{\circ} \mathrm{C}$. Moreover, Bungert et al. (1997) investigated monodisperse and bidisperse polystyrene (PS) + cyclohexane + carbon dioxide systems at high temperatures and pressures. A bidisperse PS was prepared by mixing two monodisperse PSs at a ratio of $1: 1$. The results indicated that most of the long-chain PSs were in a polymer-rich phase whereas most short-chain PSs were in a solvent-rich phase at high pressure. When lowering the pressure, long-chain PSs were not observed in the solvent-rich phase. Because the PS composition of the solvent-rich phase was low and most PS is in the PS-rich phase regardless of pressure, the molecular weight distribution (MWD) of PS in the PS-rich phase did not depend on the pressure.

Although the PE produced by the solution polymerization was used for versatile application, and a lot of LL phase boundary data have been reported so far, experimental LL phase equilibrium data have not been reported as far as the authors are aware. Therefore, in the present work, both the LL phase boundary and phase equilibria for the hexane+polydisperse PE system were measured at $473 \mathrm{~K}$ and at high pressure to elucidate and accumulate the phase equilibria for the polydisperse PE solution at high temperature and pressure. Moreover, the experimental results of phase equilibrium compositions and the MWDs of the $\mathrm{PE}$ in each phase were compared with the predicted results obtained using the Sanchez-Lacombe equation of state (S-L EOS) (Sanchez and Lacombe, 1976, 1978) in order to clarify the relationship between the operating conditions and the LL phase equilibria.

\section{Experimental}

\subsection{Materials}

Polydisperse PE that was used for the main phase equilibrium measurements was purchased from Sigma-Aldrich Corp. The MWD was determined via a gel permeation

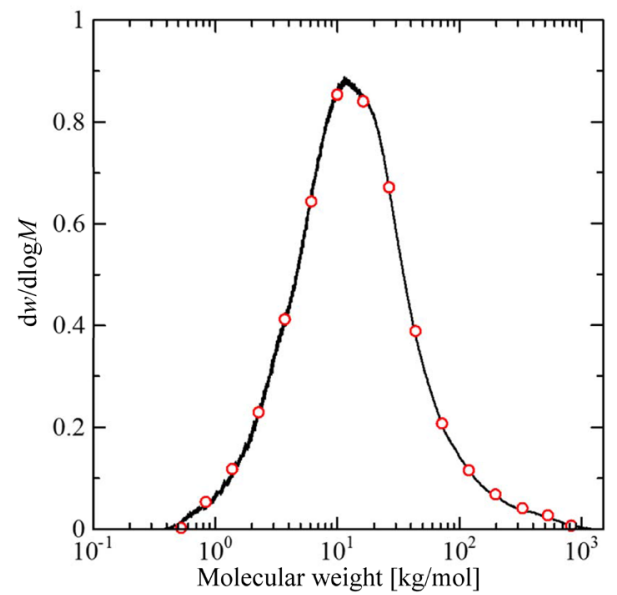

Fig. 2 MWD of PE that was used; black solid line shows the molecular weight distribution obtained via GPC; red open circles show the 16 pseudo-components of polydisperse PE used for the calculations by the S-L EOS

chromatography (GPC) system (HLC-8321 GPC/HT, Tosoh Corp.) with three columns (one was TSKgel GMH6-HTL and the other two were TSKgel GMHHR-H HT, Tosoh Corp.) that were serially cascaded. The MWD of the polydisperse PE that was calibrated using monodisperse PS standards is shown in Figure 2. The number average molecular weight $\left(\bar{M}_{\mathrm{n}}\right)$, weight average molecular weight $\left(\bar{M}_{\mathrm{w}}\right)$, and polydispersity index $\left(\bar{M}_{\mathrm{w}} / \bar{M}_{\mathrm{n}}\right)$ were $7.07 \mathrm{~kg} / \mathrm{mol}, 30.0 \mathrm{~kg} / \mathrm{mol}$, and 4.24, respectively. Hexane was also purchased from Sigma-Aldrich Corp., and its purity was $>99 \mathrm{~mol} \%$. $o$ Dichlorobenzene with purity $>98 \mathrm{~mol} \%$ was purchased from Kishida Chemical Co. and used for the mobile phase of the GPC analysis.

\subsection{Experimental procedure for LL phase boundary measurement}

The LL phase boundary curve in a wide range of PE weight fractions is also important for the investigation of LL phase equilibria, because the phase equilibrium curves of both phases are connected to the phase boundary, or shadow curves, as shown in Figure 1. In the region with a low PE weight fraction, the LL phase boundary curves were often determined by identification of the phase transition pressure 
at which a transparent liquid phase was changed to a cloudy phase by decreasing the pressure at constant temperature and feed composition. The details of the experimental apparatus and the measurement procedure were circumstantially described in our previous work (Haruki et al., 2008, 2009). However, the phase transition points were difficult to recognize at high $\mathrm{PE}$ weight fractions because the transition from a transparent phase to a cloudy phase proceeded in a gradual manner. Therefore, the LL phase boundaries for high PE weight fractions were determined by measuring the pressures at which the interface between the PE-rich and hexane-rich phases disappeared when the two separated phases were pressurized from low pressure, which was carried out using the same apparatus as used in the previous work. In this experiment, known amounts of hexane and PE were introduced into a variable-volume optical cell, and the mixture was well mixed at high pressure. The pressure was then decreased to a low value at which the two separated liquid phases existed, and the behavior of the interface was observed by gradually increasing the pressure. The lowest pressure at which the interface could not be observed was designated as the LL phase boundary pressure, and the accuracies of these measurements were $\pm 0.2 \mathrm{MPa}$.

\subsection{Apparatus and sampling procedure for LL phase equilibrium measurement}

A schematic diagram of the experimental apparatus developed in the present work for the phase equilibrium measurement is shown in Figure 3. The apparatus mainly consisted of a variable-volume optical cell $(20 \mathrm{~mm}$ in internal diameter, Nekken Co.), a pressure control part, an observation system that is often used for the apparatus to measure

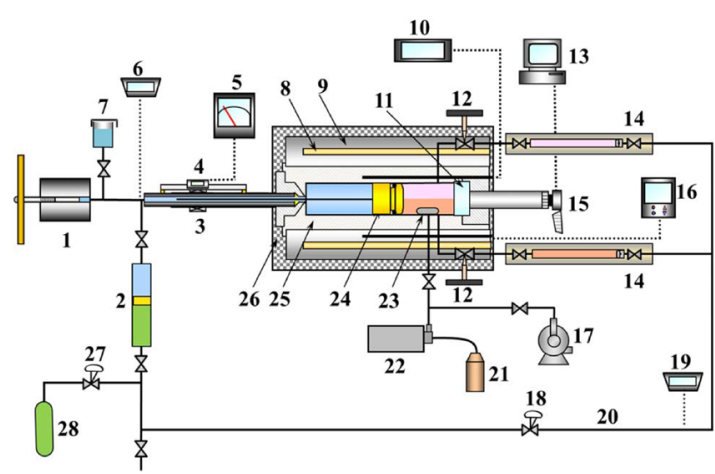

Fig. 3 Experimental apparatus for the phase equilibrium measurement; 1 . hand pump; 2. Oil/ $\mathrm{N}_{2}$ balancer; 3. linear variable differential transformer; 4 . linear scale; 5 . displacement meter; 6. pressure gauge; 7. silicone oil reservoir; 8 . cartridge heaters; 9. aluminum block; 10. thermometer and temperature indicator; 11. quartz window; 12. needle valves; 13 . personal computer; 14. sampling tubes surrounded by aluminum blocks with heaters; 15 . borescope with CCD camera; 16 . temperature controller and indicator; 17. vacuum pump; 18 . back-pressure regulator; 19. pressure gauge for $\mathrm{N}_{2}$ gas line; 20. $\mathrm{N}_{2}$ gas line; 21. hexane flask; 22. HPLC pump; 23. stirring bar; 24 . free piston; 25. variable-volume optical cell; 26 . heat insulator; 27 . regulator; $28 . \mathrm{N}_{2}$ cylinder the cloud point via a synthetic method, and a sampling system that makes it possible to obtain the compositions of two phases. The sampling tubes and the lines between the cell and the tube were heated by mantle heaters to avoid phase separation in the lines.

The internal volume of the cell could be varied up to approximately $50 \mathrm{~cm}^{3}$ by changing the position of a free piston. The cell was covered with an aluminum block, and four cartridge heaters (Sakaguchi E. H Voc Corp.) were arranged at the aluminum block to eliminate the temperature gradient inside the cell. The PE solution was agitated by a magnetic stirring bar that was coated with Teflon to avoid a metastable state. The cell temperature was measured using a platinum resistance temperature detector (NR-350, Netsushin Co.) and a temperature indicator (Model 1502 A, uncertainty $0.012 \mathrm{~K}$, Hart Scientific Co.). The pressure in the cell was controlled with a hand pump (High Pressure Equipment Co.) and measured with a pressure transmitter (PTX 600 series, uncertainty $\pm 0.08 \%$, GE Sensing and Inspection Technologies Co.) and an indicator (DPI 282, uncertainty $\pm 0.05 \%$, GE Sensing and Inspection Technologies Co.). The phase behaviors inside the optical cell were observed using a borescope (R100-038-000-50, Olympus Corp.) equipped with a CCD camera (TNC4604), ELMO Co.). The sampling tubes (SUS316 tubes, $7 \mathrm{~mm}$ in diameter and $170 \mathrm{~mm}$ in length) for each phase had a free piston inside. During the experiment, one side of the sampling tube was filled with nitrogen gas, and the pressure of the nitrogen gas was controlled by a back-pressure regulator to avoid an undesirable pressure reduction during sampling.

For the measurements, PE particulates were weighed using an electronic balance (ME614S, uncertainty $\pm 0.1 \mathrm{mg}$, Sartorius Co.), and then they were introduced into the optical cell. Once the inside of the cell was evacuated, about $17 \mathrm{~g}$ of hexane was introduced into the cell via an HPLC pump (PU-2080-CO2 Plus, Jasco Corp.). The total weights of the introduced hexane and PE were about $18-22 \mathrm{~g}$ in most measurements, and the uncertainty of the weight fraction for the mixture was estimated to be within \pm 0.002 . The temperature of the PE solution in the cell was raised to the experimental level, and a transparent liquid phase was formed at high pressure. The pressure was then decreased gradually under a constant temperature. The transparent phase became cloudy at the LL phase boundary pressure, and the two separated phases appeared. After the pressure reached the objective level and the separated phases were well agitated, the agitation inside the cell was then stopped in order to obtain stable separated phases. The needle valves at the sampling lines between the cell and the sampling tube were opened after the free pistons in each sampling tube were moved to the cell side by introducing nitrogen gas into the sampling tube at a slightly higher pressure than that inside the cell. The portion of each phase inside the cell was then slowly injected into the sampling tube by carefully operating a hand pump, a balancer, and a pressure regulator of the nitrogen gas line. In the sampling operation, one phase was first sampled in the tube, and another phase was then 
sampled to reduce the pressure fluctuation.

\subsection{Determination of the composition and the molecular weight distribution of PE for LL phase equilibrium measurements}

In the present work, the compositions of each phase were determined via a gravimetric method. The masses of the sampling tubes containing the hexane $+\mathrm{PE}$ mixture were measured using the electronic balance after they were detached from the apparatus. The tubes were then soaked in liquid nitrogen for about $5 \mathrm{~min}$ to freeze the mixtures inside because the pressure inside the tubes had to be decreased before the stop valves of the sampling tubes were opened in order to gradually evaporate the hexane. Next, the sampling tubes were heated in a step-by-step fashion to $403 \mathrm{~K}$, and the hexane was then completely evaporated by drying in a vacuum oven. The masses of the sampling tubes containing only PE were measured again, and the amount of sampled PE was estimated from the difference in the masses of the sampling tubes between before the experiment and after the drying operation. The amount of sampled hexane was also obtained from the evaporated hexane. Moreover, the MWD of PE in each phase was determined via GPC analysis.

\section{Calculation Model}

The S-L EOS was used to correlate the LL phase boundary and to predict the LL phase equilibria. The S-L EOS is expressed as follows (Sanchez and Lacombe, 1976, 1978).

$$
\begin{aligned}
& \tilde{\rho}^{2}+\tilde{P}+\tilde{T}\left[\ln (1-\tilde{\rho})+\left(1-\frac{1}{r}\right) \tilde{\rho}\right]=0 \\
& \tilde{P}=\frac{P}{P^{*}}, \quad \tilde{\rho}=\frac{\rho}{\rho^{*}}, \quad \tilde{T}=\frac{T}{T^{*}}, \quad r=\frac{M P^{*}}{R T^{*} \rho^{*}}
\end{aligned}
$$

Where, $P^{*}, \rho^{*}$ and $T^{*}$ are characteristic parameters of the S-L EOS, $r$ is the segment number: the number of lattice sites occupied by molecules. $M$ and $R$ are the molecular weight and universal gas constant, respectively. For $m$ component mixtures, the mixing rules of the characteristic parameters are given by the following equations.

$$
\begin{aligned}
& P^{*}=\sum_{i=1}^{m} \phi_{i} P_{i}^{*}-\frac{1}{2} \sum_{i=1}^{m} \sum_{j=1}^{m} \phi_{i} \phi_{j} \Delta P_{i j}^{*} \\
& \Delta P_{i j}^{*}=P_{i}^{*}+P_{j}^{*}-2\left(1-k_{i j}\right) \sqrt{P_{i}^{*} P_{j}^{*}} \\
& T^{*}=P^{*} \sum_{i=1}^{m}\left(\frac{\phi_{i}^{0} T_{i}^{*}}{P_{i}^{*}}\right) \\
& \frac{1}{r}=\sum_{i=1}^{m}\left(\frac{\phi_{i}^{0}}{r_{i}^{0}}\right) \\
& \phi_{i}^{0}=\frac{\left(\phi_{i} P_{i}^{*} / T_{i}^{*}\right)}{\sum_{j=1}^{m}\left(\phi_{j} P_{j}^{*} / T_{j}^{*}\right)}
\end{aligned}
$$

$$
\phi_{i}=\frac{\left(w_{i} / \rho_{i}^{*}\right)}{\sum_{j=1}^{m}\left(w_{j} / \rho_{j}^{*}\right)}
$$

Where, $w_{i}$ indicates the weight fraction of the $i$-th component and $k_{i j}$ in Eq. (4) represents the binary interaction parameter. In the calculations for polymer solutions including polydisperse polymer, the polymer was often regarded as a mixture of monodisperse polymers with different molecular weights (Koak and Heidemann, 2001; Krenz and Heidemann, 2007; Krenz et al., 2009). In the present work, the polydispersity of PE was represented as a mixture of 16 types of monodisperse PE for the LL phase boundary calculations in the same manner as with the previous work (Haruki et al., 2011). The effect of the number of the pseudo-components on the LL phase boundary and shadow curves was preliminarily investigated by comparing the results obtained using 16 pseudo-components with those of 32 pseudo-components. Both the obtained LL phase boundary curves and shadow curves agreed well regardless of the number of the discrete components. Therefore, the calculations were carried out by regarding the $\mathrm{PE}$ as a mixture of 16 types of monodisperse PE with different molecular weights. The represented 16 molecular weights, $M$, were determined by the experimental MWD obtained by the GPC analysis shown in Figure 2. The logarithmic basis of the molecular weights between the maximum and minimum values were divided into 16 sections at almost equal intervals, and the average values of each section were regarded as the represented molecular weight. The determined molecular weights of the 16 pseudo-components of polydisperse PE and their $\bar{M}_{\mathrm{n}}, \bar{M}_{\mathrm{w}}$ and $\bar{M}_{\mathrm{w}} / \bar{M}_{\mathrm{n}}$ are listed in Table $\mathbf{1}$ and shown in Figure 2. The lowest and highest molecular weights of the mix-

Table 1 Sixteen pseudo components of polydisperse PE for calculations via S-L EOS

\begin{tabular}{ccc}
\hline No. & $M[\mathrm{~kg} / \mathrm{mol}]$ & Content $[\mathrm{wt} \%]$ \\
\hline 1 & 0.533 & 0.3323 \\
2 & 0.849 & 1.0532 \\
3 & 1.396 & 2.5212 \\
4 & 2.298 & 4.9360 \\
5 & 3.760 & 8.7834 \\
6 & 6.192 & 13.9101 \\
7 & 10.094 & 18.0739 \\
8 & 16.506 & 17.9141 \\
9 & 26.846 & 14.1462 \\
10 & 44.103 & 8.3474 \\
11 & 72.793 & 4.4830 \\
12 & 120.488 & 2.5000 \\
13 & 199.293 & 1.4672 \\
14 & 331.156 & 0.8805 \\
15 & 534.139 & 0.5387 \\
16 & 831.452 & 0.1122 \\
\hline$M_{\mathrm{n}}[\mathrm{kg} / \mathrm{mol}]$ & 7.098 & \\
$M_{\mathrm{w}}[\mathrm{kg} / \mathrm{mol}]$ & 29.54 & \\
$M_{\mathrm{w}} / M_{\mathrm{n}}$ & 4.161 & \\
\hline
\end{tabular}


ture were 0.533 and $44.103 \mathrm{~kg} / \mathrm{mol}$, respectively, and the deviations of $\bar{M}_{\mathrm{n}}, \bar{M}_{\mathrm{w}}$ and $\bar{M}_{\mathrm{w}} / \bar{M}_{\mathrm{n}}$ between the created and experimental MWDs were 0.4, 1.5 and 1.9\%, respectively. As for the $k_{i j}$ in Eq. (4), the parameter between hexane and $\mathrm{PE}$ was regarded as a function of the molecular weight of $\mathrm{PE}$ in order to increase the accuracy of the correlated results, because when only the effect of the molecular weight represented in Eq. (2) was used, it was insufficient to obtain satisfactory calculation accuracies of both the LL phase boundary and the critical PE weight fraction simultaneously. On the other hand, the parameters between PEs with different molecular weight were set to zero.

In the LL phase boundary calculations, the calculations were carried out at a certain feed PE concentration and temperature, $473 \mathrm{~K}$, and the MWD of the PE in the original phase was kept the same as the MWD of the feed PE. On the other hand, the composition and MWD of the PE in the newly generated phase, and the phase boundary pressure were determined via the Newton-Raphson method with the convergence conditions of equality of the chemical potential of each component in both the original and newly generated phases, $\mu_{i}^{\mathrm{O}}$ and $\mu_{i}^{\mathrm{N}}$ (shown as Eq. (9)), and with unity of the sum of the mole fractions of each component in the newly generated phase.

$$
\left|1-\frac{\mu_{i}^{\mathrm{O}}}{\mu_{i}^{\mathrm{N}}}\right| \leq \varepsilon
$$

In the present study, the value of $\varepsilon$ was set to values that ranged from $10^{-10}$ to $10^{-12}$.

As for the LL phase equilibrium calculation, a flash algorithm was used to obtain the composition of both phases at a certain feed composition, temperature and pressure. The mass balance in the system is represented by the following equation for the LL equilibrium.

$$
n^{\mathrm{F}} z_{i}=n^{\mathrm{L} 1} x_{i}^{\mathrm{L} 1}+n^{\mathrm{L} 2} x_{i}^{\mathrm{L} 2}
$$

Where, $n$ indicates the molar number, and $z$ and $x$ show the mole fractions of the feed and separated liquid phases, respectively. The superscripts F, L1 and L2 represent the feed and liquid phases of lower and heavier densities, respectively. When the molar ratio of the L1 phase to the sum of both phases, $\alpha$, and the equilibrium ratios of each of the components, $K_{i}$, are represented by Eqs. (11) and (12), respectively, the mass balance of Eq. (10) can be rewrited by Eq. (13).

$$
\begin{aligned}
& \alpha=\frac{n^{\mathrm{L} 1}}{n^{\mathrm{L} 1}+n^{\mathrm{L} 2}}=\frac{n^{\mathrm{L} 1}}{n^{\mathrm{F}}} \\
& K_{i}=\frac{x_{i}^{\mathrm{L} 1}}{x_{i}^{\mathrm{L} 2}} \\
& z_{i}=\alpha x_{i}^{\mathrm{L} 1}+(1-\alpha) x_{i}^{\mathrm{L} 2}=\left\{1+\left(K_{i}-1\right) \alpha\right\} x_{i}^{\mathrm{L} 2}
\end{aligned}
$$

Therefore, the mole fractions of each phase are shown as follows using the feed mole fraction, molar ratio and equi- librium ratio.

$$
x_{i}^{\mathrm{L} 2}=\frac{z_{i}}{1+\left(K_{i}-1\right) \alpha}, \quad x_{i}^{\mathrm{L} 1}=K_{i} x_{i}^{\mathrm{L} 2}=\frac{K_{i} z_{i}}{1+\left(K_{i}-1\right) \alpha}
$$

In the present study, $K_{i}$ and $\alpha$ were set as the variables, and they were determined using the convergence conditions of Eq. (9) (replacing $\mu_{i}^{\mathrm{O}}$ and $\mu_{i}^{\mathrm{N}}$ with $\mu_{i}^{\mathrm{L} 1}$ and $\mu_{i}^{\mathrm{L} 2}$ ) with unity of the summations of the mole fractions for both liquid phases.

\section{Results and Discussion}

\subsection{Experimental LL phase boundary pressures}

The LL phase boundary pressures measured in the present work at $473 \mathrm{~K}$ are described in Figure 4 along with the experimental liquid-liquid-vapor (LLV) phase boundary that appeared at lower pressures and was extremely close to the vapor pressure of the solvent (hexane) at $473 \mathrm{~K}$ as well as the reports of other groups (Folie and Radosz, 1995; Bungert et al., 1997). The numerical data of both the LL and LLV phase boundaries are also shown in Table 2. The maximum LL phase boundary pressure appeared at a PE weight fraction that was quite small, and the phase boundary pressure monotonically decreased with increases in the PE weight fraction that went beyond the maximum pressure. With respect to the measurements, the PE-rich phase newly appeared at the phase boundary pressure of the solution at less than approximately 0.10 of the PE weight fraction. On the other hand, the hexane-rich phase newly appeared at the phase boundary pressure at feed PE weight

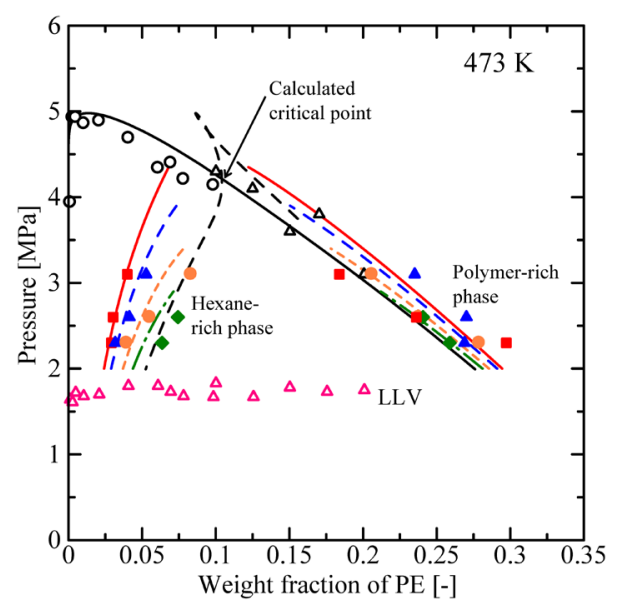

Fig. 4 Experimental and calculated LL phase boundary curve and phase equilibria for the hexane+polydisperse PE system at $473 \mathrm{~K}$; symbols $(O, \triangle)$ show the experimental LL phase boundary curves obtained by the observations of the cloudy phase appearance and by the measurements of the interface elimination, respectively; symbols $(\boldsymbol{\square}, \boldsymbol{\Delta}, \boldsymbol{O}, \boldsymbol{\bullet})$ show the experimental phase equilibria at the feed PE weight fractions of $0.075,0.10,0.15$ and 0.20 , respectively; symbol $(\triangle)$ shows the experimental LLV phase boundary; black solid and dashed lines represent the correlated LL phase boundary curve and predicted shadow curve by the S-L EOS, respectively; red, blue, orange and green lines show the predicted phase equilibrium curves of the feed PE weight fractions of $0.075,0.10,0.15$ and 0.20 , respectively 
fractions of more than 0.125 . These results indicated that the experimental critical PE weight fraction existed somewhere between 0.10 and 0.125 at $473 \mathrm{~K}$. The critical PE weight fraction, which is determined as the cross-point of the LL phase boundary and the shadow curves, is very important for both the phase equilibrium measurement and calculation, because the LL phase equilibrium lines of the hexane-rich and the PE-rich phases connect to the LL phase boundary curve or the shadow curve based on the relationship between the feed and the critical PE weight fractions, as shown in Figure 1. When the feed PE weight fraction is less than the critical PE weight fraction, the LL phase equilibrium curves of hex-

Table 2 Experimental phase boundary pressures for the hexane $+\mathrm{PE}$ system at $473 \mathrm{~K}$

\begin{tabular}{ccc}
\hline Feed PE [wt frac $]$ & L-LL ${ }^{\mathrm{a})}[\mathrm{MPa}]$ & $\mathrm{LL}^{\mathrm{L}} \mathrm{LLV}^{\mathrm{b})}[\mathrm{MPa}]$ \\
\hline 0.0011 & 3.94 & 1.64 \\
0.0027 & 4.93 & 1.61 \\
0.0049 & 4.93 & 1.72 \\
0.0103 & 4.86 & 1.68 \\
0.0208 & 4.89 & 1.70 \\
0.0407 & 4.69 & 1.80 \\
0.0608 & 4.34 & 1.80 \\
0.0694 & 4.40 & 1.73 \\
0.0781 & 4.21 & 1.68 \\
0.0984 & 4.14 & 1.67 \\
0.1001 & $4.3^{\mathrm{c})}$ & 1.83 \\
0.1250 & $4.1^{\mathrm{c})}$ & \\
0.1256 & & 1.67 \\
0.1503 & $3.6^{\mathrm{c})}$ & 1.78 \\
0.1702 & $3.8^{\mathrm{c})}$ & 1.73 \\
0.1756 & & 1.75 \\
0.2007 & $3.1^{\mathrm{c})}$ & \\
0.2009 & & \\
\hline
\end{tabular}

a) Liquid-liquid phase boundary; b) liquid-liquid-vapor phase boundary; ${ }^{c)}$ determined from disappearance of the phase interface ane- and PE-rich phases connect to the phase boundary and the shadow curves, respectively. Conversely, when the feed $\mathrm{PE}$ weight fraction is higher than that at the critical point, the hexane- and PE-rich phase lines connect to the shadow and the phase boundary curves, respectively.

\subsection{Experimental LL phase equilibria}

Before the phase equilibrium measurements, homogeneous PE solutions were sampled from both the bottom and the top of the cell into sampling tubes, and the compositions were analyzed to validate the experimental apparatus, and the sampling and analysis composition methods for the phase equilibrium measurement. These examinations were carried out using another polydisperse PE with $\bar{M}_{\mathrm{w}}=11.4 \mathrm{~kg} / \mathrm{mol}$ and $\bar{M}_{\mathrm{w}} / \bar{M}_{\mathrm{n}}=2.61$, at a temperature of $453 \mathrm{~K}$ and a pressure of $3.1 \mathrm{MPa}$ with $\mathrm{PE}$ weight fractions of 0.0099 and 0.1485 , respectively. The details of the experimental results are shown in Table 3. The PE weight fractions of the solutions sampled from both positions of the cell were very close to the feed $\mathrm{PE}$ weight fractions regardless of whether they were high or low. Therefore, the experimental apparatus, and the sampling and analysis methods used in the present work were considered to be appropriate for the LL phase equilibrium measurements of polymer solutions at high temperature and pressure.

The LL phase equilibrium measurements were carried out using PE with $\bar{M}_{\mathrm{w}}=30.0 \mathrm{~kg} / \mathrm{mol}$ and $\bar{M}_{\mathrm{w}} / \bar{M}_{\mathrm{n}}=4.24$, as shown in Figure 2. The experimental results of the relationships between the feed PE weight fractions, pressure and the LL phase equilibrium compositions for feed PE weight fractions of $0.075,0.10,0.15$, and 0.20 at $473 \mathrm{~K}$ are shown in Figure 4, and the numerical data are also listed Table 4 . The experimental results of the hexane-rich phase showed that the PE weight fraction decreased as the feed PE weight fraction and the pressure decreased. As for the PE-rich phase, the data indicated that the PE weight fraction decreased as

Table 3 Results of the test examinations based on the PE weight fractions in the solutions sampled from the top and bottom positions for the hexane+ PE system at $453 \mathrm{~K}$ and $3.1 \mathrm{MPa}$ (homogeneous phase)

\begin{tabular}{|c|c|c|c|c|}
\hline \multirow{2}{*}{$\begin{array}{c}\text { Feed } w_{\mathrm{PE}}^{\text {a) }} \\
\text { Sampling position }\end{array}$} & \multicolumn{2}{|c|}{0.0099} & \multicolumn{2}{|c|}{0.1485} \\
\hline & Top & Bottom & Top & Bottom \\
\hline Hexane in sampling tube $[\mathrm{g}]$ & 3.3491 & 4.1725 & 3.5570 & 3.6196 \\
\hline$w_{\mathrm{PE}}$ in sampling tube & 0.0097 & 0.0102 & 0.1470 & 0.1498 \\
\hline Deviation $^{\text {b) }}$ & -0.0002 & 0.0003 & -0.0015 & 0.0013 \\
\hline
\end{tabular}

${ }^{\text {a) }}$ weight fraction of $\mathrm{PE}$; ${ }^{\mathrm{b})}\left[w_{\mathrm{PE}}\right.$ in sampling tube $]-\left[w_{\mathrm{PE}}\right.$ of feed $]$

Table 4 Experimental PE weight fractions of the LL phase equilibria for the hexane $+\mathrm{PE}$ system at $473 \mathrm{~K}$

\begin{tabular}{|c|c|c|c|c|c|c|c|c|}
\hline \multirow{2}{*}{$\begin{array}{c}\text { Feed } w_{\mathrm{PE}} \\
\mathrm{P} \\
{[\mathrm{MPa}]}\end{array}$} & \multicolumn{2}{|c|}{0.075} & \multicolumn{2}{|c|}{0.10} & \multicolumn{2}{|c|}{0.15} & \multicolumn{2}{|c|}{0.20} \\
\hline & $\begin{array}{l}\text { H-rich }{ }^{\text {a) }} \\
\text { [wt frac] }\end{array}$ & $\begin{array}{l}\text { PE-rich } \\
\text { [wt frac] }\end{array}$ & $\begin{array}{l}\text { H-rich }{ }^{a)} \\
\text { [wt frac] }\end{array}$ & $\begin{array}{l}\text { PE-rich } \\
\text { [wt frac] }\end{array}$ & $\begin{array}{l}\text { H-rich }{ }^{a)} \\
\text { [wt frac] }\end{array}$ & $\begin{array}{l}\text { PE-rich } \\
\text { [wt frac] }\end{array}$ & $\begin{array}{l}\text { H-rich }{ }^{\mathrm{a})} \\
{[\text { wt frac] }}\end{array}$ & $\begin{array}{l}\text { PE-rich } \\
\text { [wt frac] }\end{array}$ \\
\hline 2.3 & 0.029 & 0.30 & 0.032 & 0.27 & 0.039 & 0.28 & 0.064 & 0.26 \\
\hline 2.6 & 0.030 & 0.24 & 0.041 & 0.27 & 0.055 & 0.24 & 0.074 & 0.24 \\
\hline 3.1 & 0.040 & 0.18 & 0.053 & 0.24 & 0.083 & 0.21 & & \\
\hline
\end{tabular}

a) Hexane-rich 
the PE weight fraction of the feed solution increased, and the PE weight fraction increased as the pressure decreased, although the dependency of the feed PE weight fraction was scattered compared with those of the hexane-rich phase. These trends of the LL phase equilibria corresponded with those reported in the review article by Folie and Radosz (1995). The reasons for the data scattering found in the PE-rich phase would be that the viscosities of the PE-rich phase were high, and it would make difficult to accurately collect the samples into the sampling tube. Moreover, at a feed PE weight fraction of 0.075 , the position of the interface between both phases was at a very low level (very small amount of PE-rich phase), which might have led to a commingling of the hexane-rich phase with the PE-rich phase during the sampling of the PE-rich phase.

\subsection{Prediction using S-L EOS}

Before the predictions for the LL phase equilibria, the LL phase boundary curve was correlated at $473 \mathrm{~K}$ via the S-L EOS. Since the phase equilibrium curves connect with the phase boundary curve or shadow curve, as shown in Figure 1, an accurate calculation of the critical point is as important as that of the phase boundary curve for accuracy of the phase equilibrium calculation. In the correlation, the characteristic parameters, $T^{*}, P^{*}$ and $\rho^{*}$ previously reported in the literature (Sanchez and Lacombe, 1976; Kiran et al., 1993b) were first used for both hexane and PE, and only the binary interaction parameter, $k_{i j}$, of the hexane-PE pair was adjusted as a fitting parameter. However, both the phase boundary curve and the critical point depend on the interaction parameter, and it was difficult to simultaneously obtain reasonable correlation results. Therefore, the $\rho^{*}$ for PE was treated as the fitting parameter as well as the $k_{i j}$ of the hexane-PE pair. The determined $\rho^{*}$ of PE was $1,200 \mathrm{~kg} / \mathrm{m}^{3}$, and is listed in Table 5 along with the characteristic parameters cited from the literature. The effect of $\rho^{*}$ on the critical point is discussed in the Appendix. As for the interaction parameter, an equation with a dependency on the molecular weight was used for the hexane-PE pairs as follows.

$$
k_{\text {hex }-\mathrm{PE}_{i}}=0.029-6.0 \times 10^{-3} \times \log \left(M_{i}[\mathrm{~kg} / \mathrm{mol}]\right)
$$

On the other hand, the interaction parameters between PEs with different molecular weights were set to zero, as described in the previous section, "2. Calculation Model".

The correlated LL phase boundaries of the hexane+polydisperse $\mathrm{PE}$ system are shown in Figure 4 along with the experimental phase boundary data. The correlated results reproduced the experimental phase bound-

Table 5 Characteristic parameters of the S-L EOS for hexane and PE

\begin{tabular}{lccc}
\hline Substance & $P^{*}[\mathrm{MPa}]$ & $\rho^{*}\left[\mathrm{~kg} / \mathrm{m}^{3}\right]$ & $T^{*}[\mathrm{~K}]$ \\
\hline Hexane & $\left.298^{\mathrm{a}}\right)$ & $775^{\mathrm{a}}$ & $476^{\mathrm{a}}$ \\
Polyethylene & $359^{\mathrm{b})}$ & 1200 & $521^{\mathrm{b})}$ \\
\hline
\end{tabular}

a) cited from Sanchez and Lacombe (1976); ${ }^{\text {b) }}$ cited from Kiran et al. (1993b) ary data, and the calculated critical PE weight fraction and pressure were 0.104 and $4.21 \mathrm{MPa}$, respectively, which corresponded with the experimental critical PE weight fraction (somewhere between 0.1 and 0.125 ) described in subsection, “3.1. Experimental LL phase boundary pressures". However, the calculated PE weight fractions of the shadow line appeared slightly lower than those of the hexane-rich phases of the feed PE weight fraction of 0.20 .

Moreover, the predicted results of the LL phase equilibrium curves of the PE weight fractions from 0.075 to 0.20 are also shown in Figure 4. As for the hexane-rich phase, the predicted phase equilibrium lines approximately reproduced the experimental data except for those of the feed PE of 0.20. One of the main reasons for the calculated errors observed for the feed PE of 0.20 is considered due to the relationship between the calculated shadow curve and the experimental phase equilibrium data, as described above. The predicted results showed that the $\mathrm{PE}$ weight fractions increased with increases in the feed PE weight fraction, which agreed with the experimental results. As for the PE-rich phase, the predicted results qualitatively reproduced the experimental phase equilibria, although the differences between the predicted results and the experimental results were found due to variations in the experimental data at the PE weight fraction of 0.075 . The calculated PE weight fraction was increased as both the feed PE weight fraction and the phase equilibrium pressure was decreased.

Moreover, the predicted and experimental MWDs of PE in each phase at 3.1 and $2.3 \mathrm{MPa}$ of the feed PE weight fractions of 0.10 and 0.15, are shown in Figure 5. Note that the predicted MWDs of the PE were composed of 16 types of monodisperse $\mathrm{PE}$, and the MWDs shown in the figure were generated by the spline interpolations between each value. The consistency of the mass balances of each component (16 types of PEs and hexane) of feed and of separated phases were satisfied in the calculated results by using the calculation method above. On the other hand, the values of the $\mathrm{d} w / \operatorname{dog} M$ of the experimental MWDs were rescaled, as the maximum value of the $\mathrm{d} w / \mathrm{d} \log M$ corresponded to that of the predicted result, because we could not obtain the mass of each phase experimentally by GPC analyses. As shown in the figure, the predicted results reproduced the experimental results, although over- and under-estimations were observed for some portions of the MWDs. The high molecular weight fractions decreased in the hexane-rich phase and the PE amounts increased in the PE-rich phase as the phase equilibrium pressure was decreased regardless of the feed $\mathrm{PE}$ weight fractions. Furthermore, the predicted results of the pressure dependencies of $\bar{M}_{\mathrm{w}}$ and $\bar{M}_{\mathrm{w}} / \bar{M}_{\mathrm{n}}$ in a wide range are described in Figure 6 along with the experimental results. As for the $\bar{M}_{w}$, the predicted results agreed well with the experimental results in the hexane-rich phase. The $\bar{M}_{\mathrm{w}}$ was increased with increasing pressure in the hexane-rich phase, and the dependency of the feed PE weight fraction was small at relatively low pressures. With respect to the PE-rich phase, although the deviations between the predicted and experimental $\bar{M}_{\mathrm{w}}$ were larger than those of the hexane-rich 

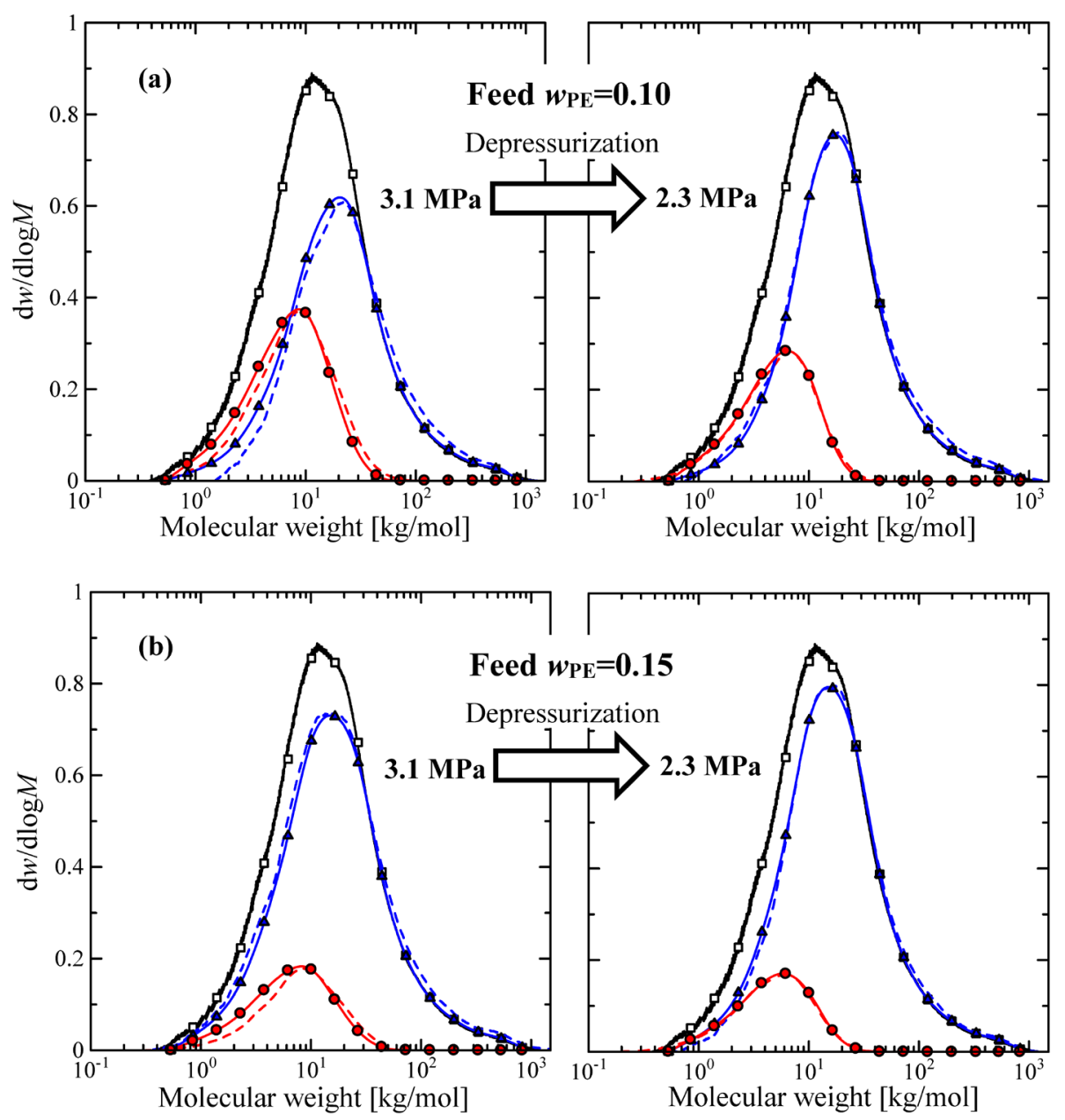

Fig. 5 Predicted and experimental MWDs of PE in each phase at the feed PE weight fractions of 0.10 and 0.15 ; (a) and (b) show the MWDs at 3.1 and 2.3 MPa and at the feed PE weight fractions 0.10 and 0.15 , respectively; black solid line shows the MWD of the feed PE obtained via GPC, and open tetragons on the feed MWD line indicate the feed 16 pseudo-components used in the calculations; solid blue and red lines with triangle and circle symbols represent the predicted MWDs for PE-rich and hexane-rich phases, respectively; dashed blue and red lines show the experimental MWDs for PE-rich and hexane-rich phases, respectively
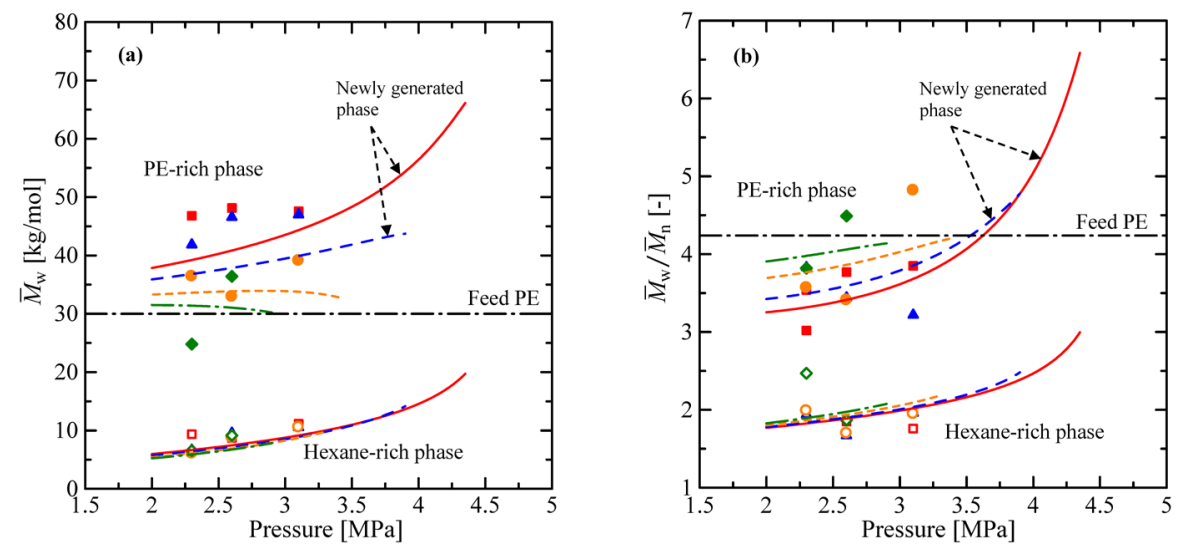

Fig. 6 Experimental results and prediction of the pressure and the feed PE weight fraction dependencies of the $\bar{M}_{\mathrm{w}}$ and $\bar{M}_{\mathrm{w}} / \bar{M}_{\mathrm{n}}$ of the PE in both the hexane- and PE-rich phases for the hexane+polydisperse PE system at $473 \mathrm{~K}$; (a) and (b) show the $\bar{M}_{\mathrm{w}}$ and $\bar{M}_{\mathrm{w}} / \bar{M}_{\mathrm{n}}$, respectively; symbols $(\square, \Delta, \diamond, \diamond)$ and $(\square, \triangle, \bigcirc, \diamond)$ show the experimental results in PE-rich and hexane-rich phases obtained at the feed PE weight fractions of $0.075,0.10,0.15$ and 0.20 , respectively; red, blue, orange and green lines show the predicted results by the S-L EOS at the feed PE weight fractions of $0.075,0.10,0.15$ and 0.20 , respectively 
phase, the predicted results qualitatively accorded with the experimental results. The dependency of the feed PE weight fraction was larger than that of the hexane-rich phase. Moreover, there were high $\bar{M}_{\mathrm{w}}$ for the feed PE weight fractions of 0.075 and 0.10 at high pressure. The reason of these behaviors is that the PEs with high-molecular weight are separated from the original phase into the new heavy phase (PE-rich phase) at the LL phase boundary pressure, and the PEs with low-molecular weight then move to the PE-rich phase as the pressure decreases.

The predicted $\bar{M}_{\mathrm{w}} / \bar{M}_{\mathrm{n}}$ approximately reproduced the experimental results in the hexane-rich phase, although some degree of difference was found between the predicted and the experimental results for the PE-rich phase, as well as for the prediction of $\bar{M}_{\mathrm{w}}$. The $\bar{M}_{\mathrm{w}} / \bar{M}_{\mathrm{n}}$ decreased with decreasing pressure both in the hexane- and PE-rich phases, and the feed PE weight fraction dependency of the $\bar{M}_{\mathrm{w}} / \bar{M}_{\mathrm{n}}$ in the PE-rich phase was larger than that in the hexane-rich phase at low pressure. Also, all the predicted curves in the hexane-rich phase were much closer regardless of the feed PE weight fraction as the same tendency as $\bar{M}_{w}$.

\section{Conclusions}

In the present study, the LL phase equilibria for the hexane+polydisperse PE system were measured at $473 \mathrm{~K}$ using an apparatus consisting of a variable-volume optical cell with sampling lines for each phase. The measured phase equilibrium curves of the hexane-rich phase showed that the PE weight fraction was decreased as the feed PE weight fraction and pressure decreased. On the other hand, the PE weight fraction of the PE-rich phase was increased with decreases in both the feed PE weight fraction and the pressure. Moreover, the LL phase equilibria were predicted using the Sanchez-Lacombe EOS wherein the polydispersity of $\mathrm{PE}$ were expressed by the mixture of 16 types of monodisperse PE with different molecular weights. Moreover, the $\rho^{*}$ parameters of $\mathrm{PE}$ and the binary interaction parameters between hexane and each PE were determined from correlations with the LL phase boundary curve. The predicted results approximately reproduced the experimental LL phase equilibrium curves for the hexane-rich phase. Moreover, the predicted results are qualitatively in accord with the experimental results for the PE-rich phase, although some degree of deviation was quantitatively found between the predicted and the experimental results. The predicted results also reproduced the experimental data for the MWDs of PEs in the LL separated phases. Both the experimental and the predicted results also indicated that the $\bar{M}_{\mathrm{w}}$ and $\bar{M}_{\mathrm{w}} / \bar{M}_{\mathrm{n}}$ of PE in the PE-rich phase depend on the feed PE weight fractions more than those in the hexane-rich phase in low-pressure regions.

\section{Acknowledgement}

This work was financially supported by MEXT/JSPS KAKENHI Grant Number 24360321.

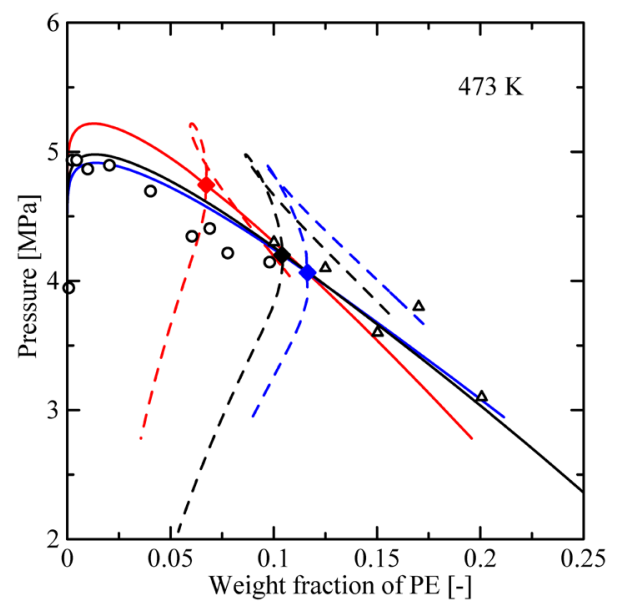

Fig. A1 The effect of $\rho^{*}$ of PE in the S-L EOS on the LL phase boundary and shadow curves for the hexane+ polydisperse PE system at $473 \mathrm{~K}$; red, black and blue solid and dashed lines show the LL phase boundary and shadow curves, respectively, obtained using $\rho^{*}$ of $895,1,200$ and $1,300 \mathrm{~kg} / \mathrm{m}^{3}$, respectively; symbols $(\diamond, \diamond, \diamond)$ indicate the calculated critical points obtained by the S-L EOS with $\rho^{*}$ of $895,1,200$ and $1,300 \mathrm{~kg} / \mathrm{m}^{3}$, respectively; symbols $(O, \triangle)$ show the experimental $\mathrm{LL}$ phase boundary curves obtained by the observations of the cloudy phase appearance and by the measurements of the interface elimination, respectively

\section{Appendix}

\section{Effect of $\rho^{*}$ of PE on the LL phase boundary and shadow curve}

The LL phase boundaries and shadow curves for the hexane + PE system at $473 \mathrm{~K}$ calculated by the S-L EOS with several values of $\rho^{*}$ of PE are illustrated in Figure A1. The other parameters used were the same as the values shown in Table 5. The interaction parameters of the hexane-PE pair represented in Eq. (15) were used. As shown in the figure, the critical points strongly depended on the $\rho^{*}$ of PE. The critical PE weight fractions and pressures were 0.067 and 4.74 MPa, 0.104 and $4.21 \mathrm{MPa}$ (as presented in 3.3 Prediction using the S-L EOS), 0.116 and $4.06 \mathrm{MPa}$ for the $\rho^{*}$ of $895,1,200$ and $1,300 \mathrm{~kg} / \mathrm{m}^{3}$, respectively. On the other hand, the dependencies of $T^{*}$ and $P^{*}$ on the critical point were not significant.

\section{Nomenclature}

$K=$ equilibrium ratio obtained by Eq. (12)

$k_{i j}=$ binary interaction parameter used in Eq. (4)

$M \quad=$ molecular weight

$\bar{M}_{\mathrm{n}} \quad=$ number average molecular weight

$\bar{M}_{\mathrm{w}} \quad=$ weight average molecular weight

$\bar{M}_{\mathrm{w}} / \bar{M}_{\mathrm{n}}=$ polydispersity index

$n=$ molar number

$P \quad=$ pressure

$P^{*} \quad=$ characteristic pressure used in S-L EOS

$\tilde{P} \quad=$ reduced pressure obtained by Eq. (2)

$=$ segment number

$=$ gas constant

[-]

[-]

$[\mathrm{kg} / \mathrm{mol}]$

$[\mathrm{kg} / \mathrm{mol}]$

$[\mathrm{kg} / \mathrm{mol}]$

[-]

[mol]

$[\mathrm{Pa}]$

[Pa]

[-]

$[-]$

$[\mathrm{J} /(\mathrm{mol} \cdot \mathrm{K})]$ 
$=$ temperature

$T^{*}=$ characteristic temperature used in S-L EOS

$\tilde{T} \quad=$ reduced temperature obtained by Eq. (2)

$w \quad=$ weight fraction

$x \quad=$ mole fraction of LL separated phases

$z \quad=$ mole fraction of feed solution

$\alpha=$ molar ratio of top phase to sum of both phases

$\varepsilon=$ threshold value for convergence of calculation used in Eq. (9)

$=$ chemical potential

$=$ density

$=$ characteristic density used in S-L EOS

$=$ reduced density obtained by Eq. (2)

$=$ close packed volume fraction in mixture

$=$ close packed volume fraction for pure component

〈Subscripts〉

$i, j \quad=$ components $i$ and $j$

〈Superscripts〉

$\mathrm{F}=$ value of feed solution

L1, L2 = values of top and bottom phases, respectively

$\mathrm{N}, \mathrm{O}=$ values of newly generated phase and original phase used in the LL phase boundary calculation (Eq. (9))

\section{Literature Cited}

Bungert, B., G. Sadowski and W. Arlt; "Supercritical Antisolvent Fractionation: Measurements in the Systems Monodisperse and Bidisperse Polystyrene-Cyclohexane-Carbon Dioxide," Fluid Phase Equilib., 139, 349-359 (1997)

Chen, S. J. and M. Radosz; "Density-Tuned Polyolefin Phase Equilibria. 1. Binary Solutions of Alternating Poly(ethylene-propylene) in Subcritical and Supercritical Propylene, 1-Butene, and 1-Hexene. Experiment and Flory-Patterson Model," Macromolecules, 25, 3089-3096 (1992)

Chen, X., K. Yasuda, Y. Sato, S. Takishima and H. Masuoka; "Measurement and Correlation of Phase Equilibria of Ethylene $+n$ Hexane+Metallocene Polyethylene at Temperatures between 373 and $473 \mathrm{~K}$ and at Pressures up to $20 \mathrm{MPa}$," Fluid Phase Equilib., 215, 105-115 (2004)

de Loos, T. W., L. J. de Graaf and J. de Swaan Arons; "Liquid-liquid Phase Separation in Linear Low Density Polyethylene-Solvent Systems," Fluid Phase Equilib., 117, 40-47 (1996)

Folie, B. and M. Radosz; "Phase Eequilibria in High-Pressure Polyethylene Technology," Ind. Eng. Chem. Res., 34, 1501-1516 (1995)

Haruki, M., Y. Takakura, H. Sugiura, S. Kihara and S. Takishima; "Phase Behavior for the Supercritical Ethylene+Hexane+Polyethylene Systems," J. Supercrit. Fluids, 44, 284-293 (2008)

Haruki, M., K. Sato, S. Kihara and S. Takishima; "High Pressure Phase Behavior for the Supercritical Ethylene+Cyclohexane+Hexane+
Polyethylene Systems," J. Supercrit. Fluids, 49, 125-134 (2009)

Haruki, M., S. Mano, Y. Koga, S. Kihara and S. Takishima; "Phase Behaviors for the Supercritical Ethylene+1-Hexene+Hexane+Polyethylene Systems at Elevated Temperatures and Pressures," Fluid Phase Equilib., 295, 137-147 (2010)

Haruki, M., K. Nakanishi, S. Mano, S. Kihara and S. Takishima; "Effect of Molecular Weight Distribution on the Liquid-Liquid Phase Separation Behavior of Polydispersed Polyethylene Solutions at High Temperatures," Fluid Phase Equilib., 305, 152-160 (2011)

Kiran, E., W. Zhuang and Y. L. Sen; "Solubility and Demixing of Polyethylene in Supercritical Binary Fluid Mixtures: Carbon DioxideCyclohexane, Carbon Dioxide-Toluene, Carbon Dioxide-Pentane," J. Appl. Polym. Sci., 47, 895-909 (1993a)

Kiran, E., Y. Xiong and W. Zhuang; "Modeling Polyethylene Solutions in Near and Supercritical Fluids Using the Sanchez-Lacombe Model," J. Supercrit. Fluids, 6, 193-203 (1993b)

Koak, N. and R. A. Heidemann; "Phase Boundary Calculations for Solutions of a Polydisperse Polymer," AIChE J., 47, 1219-1225 (2001)

Krenz, R. A. and R. A. Heidemann; "Modelling the Fluid Phase Behaviour of Polydisperse Polyethylene Blends in Hydrocarbons Using the Modified Sanchez-Lacombe Equation of State," Fluid Phase Equilib., 262, 217-226 (2007)

Krenz, R. A., T. Laursen and R. A. Heidemann; “The Modified SanchezLacombe Equation of State Applied to Polydisperse Polyethylene Solutions," Ind. Eng. Chem. Res., 48, 10664-10681 (2009)

McClellan, A. K., E. G. Bauman and M. A. McHugh; "Polymer Solution-Supercritical Fluid Phase Behavior," Supercritical Fluid Technology, pp. 161-178, J. M. L. Penninger, M. Radosz, M. A. McHugh and V. J. Krukonis eds., Elsevier Science Publishers, Amsterdam, the Netherlands (1985)

McHugh, M. A. and T. L. Guckes; "Separating Polymer Solutions with Supercritical Fluids," Macromolecules, 18, 674-680 (1985)

Nagy, I., T. W. de Loos, R. A. Krenz and R. A. Heidemann; "High Pressure Phase Equilibria in the Systems Linear Low Density Polyethylene $+n$-Hexane and Linear Low Density Polyethylene $+n$ Hexane+Ethylene: Experimental Results and Modelling with the Sanchez-Lacombe Equation of State," J. Supercrit. Fluids, 37, 115124 (2006)

Nagy, I., R. A. Krenz, R. A. Heidemann and T. W. de Loos; "HighPressure Phase Equilibria in the System Linear Low Density Polyethylene+Isohexane: Experimental Results and Modelling," J. Supercrit. Fluids, 40, 125-133 (2007)

Sanchez, I. C. and R. H. Lacombe; "An Elementary Molecular Theory of Classical Fluids. Pure Fluids," J. Phys. Chem., 80, 2352-2362 (1976)

Sanchez, I. C. and R. H. Lacombe; "Statistical Thermodynamics of Polymer Solutions," Macromolecules, 11, 1145-1156 (1978)

Seckner, A. J., A. K. McClellan and M. A. McHugh; "High-Pressure Solution Behavior of the Polystyrene-Toluene-Ethane System," AIChE J., 34, 9-16 (1988)

Xiong, Y. and E. Kiran; "High-Pressure Phase Behavior in Polyethylene/ $n$-Butane Binary and Polyethylene/n-Butane $/ \mathrm{CO}_{2}$ Ternary Systems," J. Appl. Polym. Sci., 53, 1179-1190 (1994) 